Received: 1 March 2019

Accepted: 28 June 2019

Published online: 12 July 2019


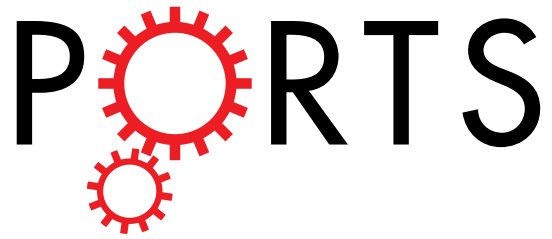

\title{
OPEN
}

\section{Incremental Peritoneal Dialysis} May be Beneficial for Preserving Residual Renal Function Compared to Full-dose Peritoneal Dialysis

Yeonhee Lee ${ }^{1}$, Sung Won Chung $\mathbb{1}^{1}$, Seokwoo Park ${ }^{1}$, Hyunjin Ryu ${ }^{1}$, Hajeong Lee ${ }^{1}$, Dong Ki Kim $\mathbb{D}^{1}$, Kwon Wook Joo ${ }^{1}$, Curie Ahn $\mathbb{(}^{1}{ }^{1}$, Joongyub Lee ${ }^{2}$ \& Kook-Hwan Oh $\oplus^{1}$

Maintaining residual renal function (RRF) is a crucial issue in peritoneal dialysis (PD). Incremental dialysis is the practice of initiating PD exchanges less than four times a day in consideration of RRF, and increasing dialysis dose in a step-wise manner as the RRF decreases. We aimed to compare the outcomes of incremental PD and full-dose PD in terms of RRF preservation and other outcomes. This was a single-center, observational study. Data were extracted retrospectively from a cohort of incident PD patients over 16 years old who started PD between 2007 and 2015 in the PD Unit of Seoul National University Hospital. We used inverse probability weighting (IPW) adjustment based on propensity scores to balance covariates between the incremental and full-dose PD groups. Multivariate, timedependent Cox analyses were performed. Among 347 incident PD patients, 176 underwent incremental PD and 171 underwent conventional full-dose PD. After IPW adjustment, the incremental PD group exhibited a lower risk of developing anuria (hazard ratio [HR] 0.61, 95\% confidence interval [CI] $0.43-$ 0.88). Patient survival, technique survival, and peritonitis-free survival were all similar between these groups ( $P>0.05$ by log-rank test). Incremental PD was beneficial for preserving RRF and showed similar patient survival when compared to conventional full-dose PD.

Maintaining residual renal function (RRF) is a crucial issue in patients with end-stage renal disease (ESRD), because it has been reported in numerous observational studies to play an important role in the adequacy of dialysis, quality of life, and patient survival in dialysis patients ${ }^{1-7}$. A higher RRF is associated with better patient survival in both peritoneal dialysis (PD) and hemodialysis (HD) patients ${ }^{8,9}$. PD is the preferred renal replacement modality for the preservation of $\mathrm{RRF}^{10}$; in particular, the rate of RRF decline was slower in patients receiving PD than in HD patients ${ }^{11,12}$. Moreover, PD can slow the decline of RRF compared to the natural slope of RRF decline prior to dialysis initiation ${ }^{13,14}$.

Incremental PD is the practice of initiating PD exchanges less than four times a day in consideration of RRF, and increasing dialysis dose in a step-wise manner as the RRF decreases, by achieving a minimum weekly Kt/V target of 1.7 as suggested by the NKF-KDOQI (National Kidney Foundation - Kidney Disease Outcomes Quality Initiative) ${ }^{15}$ and ISPD (International society for peritoneal dialysis) 2006 Guidelines ${ }^{16}$ and not falling below the acceptable minimum. This gradual initiation of dialysis may make the process of dialysis less invasive and better tolerated hemodynamically, as well as allowing for a greater adaptation to patients ${ }^{17,18}$.

Continuous ambulatory peritoneal dialysis (CAPD) may be an ideal method for beginning an incremental dialysis strategy. Starting dialysis gradually, with 1 to 2 manual exchanges a day and graduating up to a maximal 4 exchanges a day (full-dose) if needed, could be an acceptable method to more gently transition into renal replacement therapy, and is better tolerated by patients in terms of lifestyle disruption.

Not only did more frequent and longer dialysis not improve clinical outcomes in either PD or HD patients, randomized controlled trials have also shown inconsistent results ${ }^{19-22}$. Indeed, more frequent and lengthy dialysis may even accelerate RRF decline ${ }^{23}$. Moreover, previous studies have suggested that an incremental approach to PD initiation has some advantages associated with the lower number of exchanges per session, such as a lower

${ }^{1}$ Department of Internal Medicine, Seoul National University College of Medicine, Seoul, Korea. ${ }^{2}$ Prevention and Management Center, Inha University Hospital, Incheon, Korea. Correspondence and requests for materials should be addressed to J.L. (email: tp240@naver.com) or K.-H.O. (email: khoh@snu.ac.kr) 
incidence of peritonitis, a better quality of life, reduced cost, and preservation of RRF ${ }^{17,24,25}$. However, effects of incremental PD on the RRF and technique survival have not been widely studied yet. The objective of this study was to compare the outcomes of incremental PD and full-dose PD in terms of RRF preservation and other PD-related outcomes.

\section{Materials and Methods}

Study design and patients. This was a single-center, retrospective cohort study. Data were extracted retrospectively from a cohort of incident PD patients who commenced PD between January 1, 2007 and December 31, 2015 in the PD Unit of Seoul National University Hospital. Patients were excluded from the study if they met any of the following criteria: total duration of PD less than 6 months, initiation of PD at another hospital, urine volume of $<200 \mathrm{~mL}$ per day at the time of initiating PD, previous hemodialysis, less than 16 years of age, and incomplete study data. Patients who continued PD were followed until July 31, 2017 and were censored at the time of death or loss to follow-up. To control for baseline comorbidities in the analysis, patient demographics, clinical, and laboratory data were recorded.

The RRF was assessed by GFR calculated as the mean of urea and creatinine clearances normalized to $1.73 \mathrm{~m}^{2}$ BSA calculated using the du Bois and du Bois formula, as follows: 0.007184 (body weight in $\mathrm{kg}$ ) ${ }^{0.425}$ (height in $\mathrm{cm})^{0.725}$. Every 6 months, including the first month after dialysis, a total weekly Kt/V was calculated relating the values taken from the 24-hour collection of urine and PD fluid to assess peritoneal and renal solute clearance was.

Operationally, full-dose PD was defined as the initiation of PD with 3 or more exchanges per day for CAPD, 7 days a week, irrespective of RRF. Incremental PD was defined as one or two dwells per day on CAPD, 7 days a week, and a peritoneal $\mathrm{Kt} / \mathrm{V}<1.7$ per week, but a total $\mathrm{Kt} / \mathrm{V} \geq 1.7$ per week ${ }^{26-28}$. However, since no patients treated with APD met the stringent criteria of incremental $\mathrm{APD}^{28}$, we analyzed data after excluding all the patients treated with APD. Also, patients who did not comply with our operational definition of full dose or incremental PD were excluded from the study. The comparison between incremental and full-dose PD approaches was based on an intention-to-treat analysis. That is, for the incremental PD group, the delivered PD dose was gradually increased to a full-dose over time, as some patients' RRF decreased. In this case, such patients were categorized as the incremental PD group.

This study was approved by the Seoul National University Hospital Institutional Review Board (No. H-1706114-860) and complied with the Declaration of Helsinki. The informed consents were waived by the IRB, because this study was a retrospective study.

Outcomes. The primary outcome of this study was time to anuria from initiation of dialysis, such that the urine volume reflected the RRF. Anuria was defined as a urine volume of $<100 \mathrm{~mL}$ per day.

Secondary outcomes were peritonitis, technique failure, and all-cause mortality. Peritonitis was diagnosed according to the 2006 guidelines from the ISPD ${ }^{29}$, and peritonitis rate was calculated as the number of peritonitis episodes per patient-year at risk. PD technique failure was defined as a transfer to hemodialysis and death directly related to PD-related complication. Causes of PD technique failure were as follows: peritonitis, inadequate PD, catheter complications, other abdominal or PD-related complications, and psychosocial barriers ${ }^{30,31}$. For analysis of time-to-technique failure, patients were censored at the time of death due to other causes, kidney transplantation, and follow-up loss. Death was determined using either hospital medical records or data from the National Database of Statistics Korea by using the Korean resident registration number.

Adjustment for differences between groups. Given that our study used non-randomized, observational data, it was anticipated that the incremental and full-dose PD groups would differ substantially with respect to characteristics at the point of dialysis initiation, because the patient's RRF may influence the selection between the two methods. The propensity score to estimate the probability, on the basis of patient characteristics, that patients would be selected for incremental PD was calculated with the use of logistic regression analyses to balance the baseline characteristics of the patients' between-group differences. Variables included in the propensity score calculation are shown in Table 1 . Then, IPW based on the propensity score was used as a tool for creating balance, with weighting each patient who underwent full-dose PD by the inverse of the probability that he or she would be selected for full-dose PD and weighting each patient who underwent incremental PD by the inverse of the probability that he or she would be selected for incremental PD. We verified the performance of the IPW procedure by comparing the distribution of covariates and standardized differences between groups both before and after IPW.

Statistical analysis. The data are described with means \pm standard deviations for continuous variables, and frequencies and proportions for categorical variables. A comparison of baseline characteristics was performed with the Wilcoxon rank-sum test for continuous variables, and the use of the Pearson chi-square test for categorical variables. We estimated Kaplan-Meier survival curves adjusted with the use of inverse probability weighting, and compared anuria-free survival between groups using the log-rank test. Since the proportional hazards assumption was not met for the incremental group variable as shown by the crossing survival curves, multivariable, time-dependent Cox proportional hazards regression analyses were performed to identify the association between incremental approach of PD and survival. We then calculated an adjusted hazard ratio and created weighted adjusted survival curves. All tests were two-sided, and $P<0.05$ was considered statistically significant. The analyses were performed with the use of the statistical software packages SPSS (version 22) and R (version 3.5.0). 


\begin{tabular}{|c|c|c|c|c|c|c|c|c|}
\hline & \multicolumn{4}{|c|}{ Unadjusted (without IPW) } & \multicolumn{4}{|c|}{ After IPW adjustment } \\
\hline & $\begin{array}{l}\text { Incremental PD } \\
(\mathrm{n}=176)\end{array}$ & $\begin{array}{l}\text { Full-dose PD } \\
(\mathrm{n}=171)\end{array}$ & $P$ value & \begin{tabular}{|l|} 
Standardized \\
Difference
\end{tabular} & $\begin{array}{l}\text { Incremental PD } \\
(\mathrm{n}=176)\end{array}$ & $\begin{array}{l}\text { Full-dose PD } \\
(\mathbf{n}=171)\end{array}$ & $P$ value & $\begin{array}{l}\text { Standardized } \\
\text { Difference }\end{array}$ \\
\hline Age (yr) & $49.2 \pm 12.63$ & $43.9 \pm 13.07$ & $<0.001$ & 0.41 & $49.2 \pm 12.63$ & $48.2 \pm 13.66$ & 0.666 & 0.08 \\
\hline Male sex (\%) & 52.3 & 52.0 & 1.000 & $<0.01$ & 52.3 & 50.3 & 0.811 & 0.04 \\
\hline BMI $\left(\mathrm{kg} / \mathrm{m}^{2}\right)$ & $22.3 \pm 3.04$ & $22.4 \pm 3.37$ & 0.699 & 0.04 & $22.3 \pm 3.04$ & $22.6 \pm 2.90$ & 0.530 & 0.10 \\
\hline Primary renal disease (\%) & & & 0.306 & 0.29 & & & 0.613 & 0.32 \\
\hline Diabetic nephropathy & 29.0 & 19.3 & & 0.22 & 29.0 & 29.0 & & 0.00 \\
\hline Hypertension & 14.8 & 21.6 & & 0.17 & 14.8 & 21.3 & & 0.16 \\
\hline Glomerulonephritis & 33.0 & 38.0 & & 0.10 & 33.0 & 27.6 & & 0.11 \\
\hline Polycystic kidney disease & 2.8 & 1.8 & & 0.06 & 2.8 & 1.7 & & 0.07 \\
\hline Graft failure & 6.2 & 4.7 & & 0.06 & 6.2 & 5.0 & & 0.05 \\
\hline Others & 5.1 & 4.7 & & 0.01 & 5.1 & 10.4 & & 0.19 \\
\hline Unknown & 9.1 & 9.9 & & 0.02 & 9.1 & 4.9 & & 0.16 \\
\hline \multicolumn{9}{|l|}{ Comorbidities (\%) } \\
\hline Hypertension & 88.1 & 84.8 & 0.463 & 0.09 & 88.1 & 89.0 & 0.839 & 0.03 \\
\hline Diabetes mellitus & 33.0 & 24.0 & 0.083 & 0.20 & 33.0 & 30.5 & 0.763 & 0.05 \\
\hline Visual disturbance & 19.9 & 12.3 & 0.075 & 0.20 & 19.9 & 20.8 & 0.908 & 0.02 \\
\hline Ischemic heart disease & 4.5 & 1.8 & 0.239 & 0.16 & 4.5 & 2.3 & 0.414 & 0.12 \\
\hline Cerebrovascular disease & 1.7 & 2.3 & 0.969 & 0.04 & 1.7 & 0.9 & 0.444 & 0.06 \\
\hline Peripheral vascular disease & 5.1 & 2.9 & 0.445 & 0.11 & 5.1 & 1.8 & 0.093 & 0.18 \\
\hline Congestive heart failure & 7.4 & 3.5 & 0.177 & 0.17 & 7.4 & 2.4 & 0.063 & 0.23 \\
\hline Hepatitis & 6.2 & 5.3 & 0.870 & 0.04 & 6.2 & 10.5 & 0.400 & 0.15 \\
\hline Malignancy & 3.4 & 2.3 & 0.784 & 0.06 & 3.4 & 2.6 & 0.720 & 0.04 \\
\hline Kidney transplantation & 8.0 & 4.7 & 0.302 & 0.13 & 8.0 & 5.0 & 0.485 & 0.12 \\
\hline \multicolumn{9}{|l|}{ Medication (\%) } \\
\hline ISA & 4.5 & 5.3 & 0.951 & 0.03 & 4.5 & 4.8 & 0.936 & 0.01 \\
\hline ACEI/ARB & 79.0 & 72.5 & 0.201 & 0.15 & 79.0 & 70.2 & 0.211 & 0.20 \\
\hline Davies comorbidity index & & & 0.04 & 0.27 & & & 0.544 & 0.13 \\
\hline 0 (Low risk) & 52.8 & 65.5 & & 0.26 & 52.8 & 59.2 & & 0.12 \\
\hline 1-2 (Medium risk) & 46.0 & 32.7 & & 0.27 & 46.0 & 40.2 & & 0.11 \\
\hline$\geq 3$ (High risk) & 1.1 & 1.8 & & 0.05 & 1.1 & 0.6 & & 0.05 \\
\hline Urine volume (mL/day) & $1576 \pm 595.2$ & $943 \pm 530.1$ & $<0.001$ & 1.12 & $1576 \pm 595.2$ & $1451 \pm 494.8$ & 0.111 & 0.22 \\
\hline Residual renal function $\left(\mathrm{mL} / \mathrm{min} / 1.73 \mathrm{~m}^{2}\right)$ & $6.9 \pm 3.00$ & $4.2 \pm 2.36$ & $<0.001$ & 1.00 & $6.9 \pm 3.00$ & $6.5 \pm 2.43$ & 0.298 & 0.16 \\
\hline Weekly peritoneal Kt/V & $1.05 \pm 0.48$ & $1.48 \pm 0.350$ & $<0.001$ & 1.01 & $1.05 \pm 0.48$ & $1.27 \pm 0.36$ & $<0.001$ & 0.52 \\
\hline Weekly renal Kt/V & $1.38 \pm 0.64$ & $0.74 \pm 0.449$ & $<0.001$ & 1.15 & $1.38 \pm 0.64$ & $1.22 \pm 0.51$ & 0.099 & 0.27 \\
\hline Total weekly Kt/V & $2.42 \pm 0.68$ & $2.22 \pm 0.531$ & 0.002 & 0.33 & $2.42 \pm 0.68$ & $2.49 \pm 0.59$ & 0.515 & 0.10 \\
\hline Peritoneal CrCl (L/week/1.73 m²) & $26.1 \pm 13.06$ & $38.0 \pm 9.68$ & $<0.001$ & 1.03 & $26.1 \pm 13.06$ & $33.9 \pm 9.54$ & $<0.001$ & 0.68 \\
\hline Total CrCl (L/week/1.73 m²) & $96.1 \pm 29.24$ & $80.7 \pm 24.31$ & $<0.001$ & 0.57 & $96.1 \pm 29.24$ & $99.4 \pm 25.25$ & 0.451 & 0.12 \\
\hline
\end{tabular}

Table 1. Baseline characteristics for the cohort before and after inverse probability weighting (IPW) adjustment. Abbreviations: PD, peritoneal dialysis; BMI, body mass index; ISA, immunosuppressive agent; ACEI, angiotensin-converting enzyme inhibitor; ARB, angiotensin II receptor blocker; $\mathrm{CrCl}$, creatinine clearance. Values are expressed as mean \pm SD or percentages. The propensity score was calculated using a logistic regression model, regressed on observed baseline characteristics (age, sex, BMI, primary renal disease, all comorbidities, history of medication such as immunosuppressive agent and ACEI or ARB, urine volume, weekly renal Kt/V, except for the variables such as residual renal function, weekly peritoneal Kt/V, total weekly $\mathrm{Kt} / \mathrm{V}$ and creatinine clearance, with which multicollinearity was detected. Peritoneal and renal solute clearances were measured at time of first month after dialysis.

\section{Results}

Population characteristics. A total of 347 incident PD patients, assessed were eligible for inclusion, started PD in the PD Unit of Seoul National University Hospital during the study period (January 1, 2007 to December 31, 2015); 176 underwent incremental PD and 171 underwent conventional full-dose PD (Fig. 1). The baseline characteristics of the patients are shown in Table 1. Before adjustment using inverse probability weighting (IPW), patients in the incremental PD group were generally older, and a higher proportion were medium-risk patients by the Davies comorbidity index, compared with patients who initiated full-dose PD. There was no significant difference in sex, the composition of primary renal disease, comorbidities, and the use of ACEI or ARB between the two groups. The baseline mean urine volume in each group were $1576 \pm 595.2 \mathrm{~mL}$ in the incremental PD group and $943 \pm 530.1 \mathrm{~mL}$ in the full-dose PD group. Peritoneal and renal solute clearances were measured between four and six weeks after initiating PD. Compared with patients initiating full-dose PD, the incremental PD group had a higher residual renal 


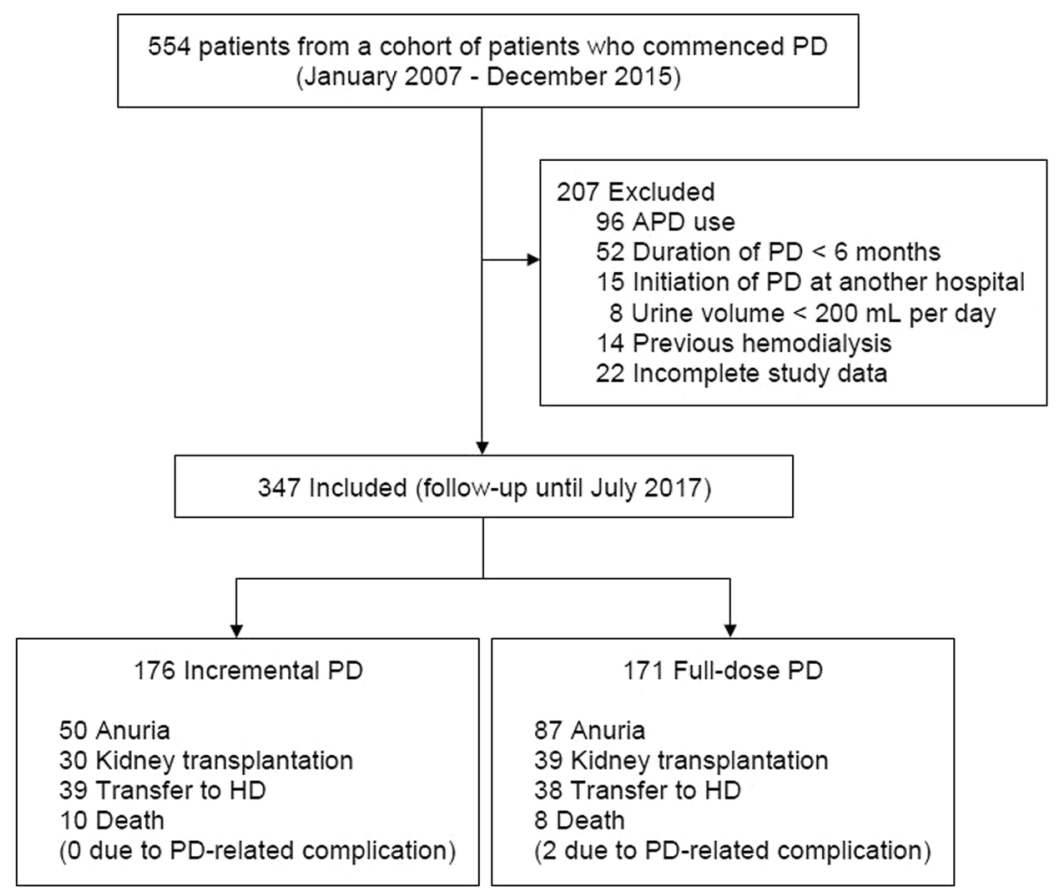

Figure 1. Flow of patients in the cohort. PD, peritoneal dialysis; HD, hemodialysis.

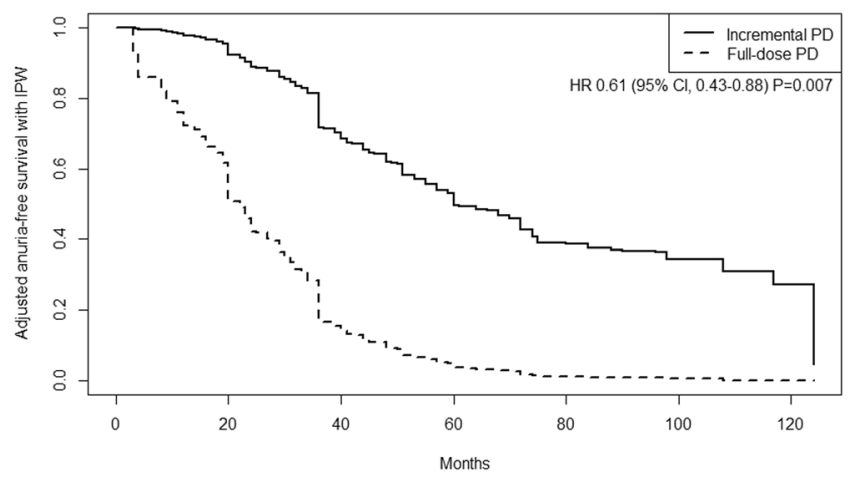

Figure 2. Inversed probability weighted, adjusted anuria-free survival using time-dependent Cox proportional hazards model.

function, weekly renal Kt/V and urine volume, whereas had a lower peritoneal $\mathrm{Kt} / \mathrm{V}$ and peritoneal $\mathrm{CrCl}$, than the full-dose PD group. For the incremental PD group, as patients' RRF decreased, the delivered PD dose gradually increased over time. The median duration of incremental PD was 2.6 years (interquartile range [IQR] 1.6-4.5 years, maximum 9.2 years). After IPW adjustment, the balance in baseline characteristics, especially the variables of renal solute clearances and urine volume between incremental and full-dose PD groups improved (Table 1).

Residual renal function - anuria-free survival. Fifty (28.4\%) patients in the incremental PD group and 87 (50.9\%) in the full-dose PD group developed anuria over time. The median follow-up duration of all patients was 5.9 years (IQR 3.3-7.8 years, maximum 10.6 years). Figure 2 shows the cumulative probability of remaining anuria-free in two groups. Incremental PD was associated with significantly lower risks of anuria (hazard ratio $[\mathrm{HR}] 0.61,95 \%$ confidence interval $[\mathrm{CI}] 0.43-0.88 ; P=0.007)$ in univariable, time-dependent Cox proportional hazards model analyses with IPW-weighted for anuria (Fig. 2). In multivariable analyses, the other independent predictors of developing anuria were younger age and history of kidney transplantation (Table 2). There was no significant association between initial urine volume and anuria.

Incidence of peritonitis and peritonitis-free survival. Both the time to the first episode of peritonitis and the incidence of peritonitis were assessed. The analysis of time-to-first peritonitis included 144 episodes of peritonitis over 1,400 patient-years. The incidence rates of first peritonitis were 0.10 episodes per patient-year (95\% CI 0.08-0.13) in the incremental PD group and 0.10 per patient-year (95\% CI 0.08-0.12) in the full-dose PD group (Table 3 ). The overall median time to the first peritonitis was 2.3 years. A Kaplan-Meier curve for the probability of remaining peritonitis-free showed no difference between the two groups $(P=0.860$ by log-rank test). 


\begin{tabular}{|l|l|c|}
\hline Variable & HR $(\mathbf{9 5} \% \mathbf{C I})$ & $\boldsymbol{P}$ value \\
\hline Incremental PD & $0.55(0.34-0.89)$ & 0.015 \\
\hline Age & $0.97(0.95-0.98)$ & $<0.001$ \\
\hline Kidney transplantation & $7.45(1.00-55.61)$ & 0.05 \\
\hline Urine volume (per 100 mL) & $0.97(0.94-1.01)$ & 0.162 \\
\hline
\end{tabular}

Table 2. Results of multivariable, time-dependent Cox proportional hazards model analyses with IPWweighted for anuria. Abbreviations: IPW, inverse probability weighting; HR, hazard ratio; CI, confidence interval; PD, peritoneal dialysis.

\begin{tabular}{|l|l|l|}
\hline \multirow{2}{*}{} & First episode \\
\cline { 2 - 3 } & $\begin{array}{l}\text { Incremental PD } \\
(\mathbf{n = 1 7 6})\end{array}$ & $\begin{array}{l}\text { Full-dose PD } \\
(\mathbf{n}=\mathbf{1 7 1})\end{array}$ \\
\hline Number of first peritonitis & 71 & 73 \\
\hline Follow-up time (patient-year) & 692.9 & 750.9 \\
\hline Peritonitis incidence (episode / patient-year) (95\% CI) & $0.10(0.08-0.13)$ & $0.10(0.08-0.12)$ \\
\hline
\end{tabular}

Table 3. Incidence of first peritonitis (144 episodes) among 347 study participants. Abbreviations: PD, peritoneal dialysis; $\mathrm{CI}$, confidence interval.



Figure 3. Kaplan-Meier survival estimates using inverse probability weighting (IPW).

Technique survival and mortality. A total of 78 (22.5\%) patients experienced PD technique failure over the follow-up period; of them, 2 patients died due to PD-related complications. When we compare the technique failure-free survival between the incremental and full-dose PD groups using the Kaplan-Meier method, it was not significantly different (The median time to the technique failure 2.7 years in the incremental PD group vs. 2.9 years in the full-dose PD group; $P=0.332$ by log-rank test).

Death from any cause occurred in 10 patients in the incremental PD group (10.9 events per 1000 person-years; 95\% CI 5.2-20.0) and 8 in the full-dose PD group (7.6 events per 1000 person-years; 95\% CI 3.3-15.0). In the log-rank test, the between-group difference was not significant $(P=0.449)$ (Fig. 3).

\section{Discussion}

The basic assumption of incremental dialysis is to reach the minimal target for adequate dialysis by summing renal function and dialysis dose. Mehrotra et al. and Golper first described an early and incremental approach to $\mathrm{PD}^{32,33}$. Early studies included only a small number of patients, and lacked control groups and any statistical comparison. De Vecchi et al. first reported working activity, degree of rehabilitation, and quality of life in incremental PD patients; quality of life and social rehabilitation were better preserved.

There is still disagreement on whether PD should begin with a full dose or with incremental doses to compensate for the amount of Kt/V no longer supplied by the RRF. Despite this consideration, incremental PD has become increasingly relevant with a world-wide trend to gradually initiate chronic dialysis at higher levels of renal function over the past 2 decades; in Italy, 29\% of patients start PD with an incremental approach, and 51\% of patients were on incremental PD at a single Canadian academic center ${ }^{17,34}$. Moreover, it is referenced as an option in a number of prominent clinical guidelines and practice recommendations. The possible benefits of incremental PD, including an improved quality of life, reduced glucose exposure to the peritoneal membrane, better peritonitis-free survival, longer preservation of RRF, and lower costs, may explain its widespread use. It is also considered an ideal bridge to renal transplantation.

This study showed that incremental PD was beneficial for preserving RRF (as demonstrated by a longer anuria-free time) compared to conventional full-dose PD, with similar technique survival, peritonitis-free survival, and mortality. The results of the study give support to the preserving effect of incremental PD on the course 
of RRF. Golper and Mehrotra recently expanded on Bricker's intact nephron hypothesis, and suggested that an incremental approach to the initiation of dialysis might help preserve RRF by both reducing nephron hyperfiltration and deactivating certain adaptive stimuli which occur in the setting of reduced nephron numbers ${ }^{35}$. Moreover, they noted that an incremental approach to the transitioning of patients from CKD to dialysis may make the patients more receptive to treatment, as it would provide a better quality of life without interfering with their daily activities.

Our findings confirm previous studies that younger age is associated with a rapid decrease in $\mathrm{RRF}^{1}$. In particular, patients initiating PD after kidney transplant failure also suffered a relatively rapid loss in $\mathrm{RRF}^{36,37}$, probably as a consequence of cessation of immunosuppression and associated inflammation ${ }^{37}$.

The strengths of this study include its relatively large cohort and inclusiveness. We included all incident CAPD patients during the study period in our PD unit. Developing a large-scale, multi-centre, randomized, controlled trials comparing incremental PD with full-dose PD is not feasible. Instead, we were able to obtain stable and reliable clinical data from a relatively large number of $\mathrm{PD}$ patients with regular monitoring of RRF and dialysis adequacy. IPW maximized data available while maintaining balance of measured covariates between groups and producing a minimally biased effect estimate ${ }^{38}$.

This study has several limitations. We acknowledge the limitations inherent to single-center experience. The study is an observational study, and the unadjusted clinical profile and propensity scores for incremental PD differed between the treatment groups. The clinician's decision between full-dose and incremental PD might be affected by a number of clinical factors such as RRF, lifestyle, body size, gender, and comorbidities. Subjects treated with APD, which accounted for $20 \%$ of total PD population were not included in the analysis, since no APD treatment in our center met the stringent criteria of incremental APD. Although we excluded patients with urine volume of $<200 \mathrm{~mL}$ per day and tried to adjust all measurable risk factors using IPW for achieving a good balance between the incremental and full-dose PD groups, the potential remains for unmeasured confounders to have influenced the findings. Also, a competing risk analysis was not conducted. Some patients died before anuria events, although these cases were appropriately censored. Lastly, the specific prescriptions of incremental PD, mainly based on the summation of peritoneal and renal small solute clearances, were inconsistent and diverse. Dialysis regimens for incremental approach have only been established in a few studies, and their successful implemental requires more clinical experience.

In conclusion, this study demonstrates that incremental PD was beneficial for preserving residual renal function (RRF) compared to conventional full-dose PD, with similar technique survival and mortality rates between the two groups. Therefore, incremental PD is a safe modality for initiating dialysis, and a longer preservation of RRF may have additional positive effect on patients who are waiting for kidney transplantation. Further prospective studies to explore the effects of incremental PD on RRF are needed.

\section{References}

1. Perl, J. \& Bargman, J. M. The importance of residual kidney function for patients on dialysis: a critical review. Am J Kidney Dis 53, 1068-1081, https://doi.org/10.1053/j.ajkd.2009.02.012 (2009).

2. Termorshuizen, F. et al. The relative importance of residual renal function compared with peritoneal clearance for patient survival and quality of life: an analysis of the Netherlands Cooperative Study on the Adequacy of Dialysis (NECOSAD)-2. Am J Kidney Dis 41, 1293-1302 (2003)

3. Termorshuizen, F. et al. Relative contribution of residual renal function and different measures of adequacy to survival in hemodialysis patients: an analysis of the Netherlands Cooperative Study on the Adequacy of Dialysis (NECOSAD)-2. J Am Soc Nephrol 15, 1061-1070 (2004).

4. Obi, Y. et al. Residual Kidney Function Decline and Mortality in Incident Hemodialysis Patients. J Am Soc Nephrol 27, 3758-3768, https://doi.org/10.1681/ASN.2015101142 (2016).

5. van der Wal, W. M. et al. Full loss of residual renal function causes higher mortality in dialysis patients; findings from a marginal structural model. Nephrol Dial Transplant 26, 2978-2983, https://doi.org/10.1093/ndt/gfq856 (2011).

6. Lopes, G. B. \& Lopes, A. A. Residual kidney function and quality of life in incident hemodialysis patients. Am J Kidney Dis 57, 179; author reply 179-180, https://doi.org/10.1053/j.ajkd.2010.09.020 (2011).

7. Liao, C. T. et al. Rate of decline of residual renal function is associated with all-cause mortality and technique failure in patients on long-term peritoneal dialysis. Nephrol Dial Transplant 24, 2909-2914, https://doi.org/10.1093/ndt/gfp056 (2009).

8. Bargman, J. M., Thorpe, K. E., Churchill, D. N. \& Group, C. P. D. S. Relative contribution of residual renal function and peritoneal clearance to adequacy of dialysis: a reanalysis of the CANUSA study. J Am Soc Nephrol 12, 2158-2162 (2001).

9. Shemin, D., Bostom, A. G., Laliberty, P. \& Dworkin, L. D. Residual renal function and mortality risk in hemodialysis patients. Am J Kidney Dis 38, 85-90, https://doi.org/10.1053/ajkd.2001.25198 (2001).

10. Vonesh, E. F., Snyder, J. J., Foley, R. N. \& Collins, A. J. Mortality studies comparing peritoneal dialysis and hemodialysis: what do they tell us? Kidney Int Suppl, S3-11, https://doi.org/10.1038/sj.ki.5001910 (2006).

11. Misra, M. et al. Effect of cause and time of dropout on the residual GFR: a comparative analysis of the decline of GFR on dialysis. Kidney Int 59, 754-763, https://doi.org/10.1046/j.1523-1755.2001.059002754.x (2001).

12. Moist, L. M. et al. Predictors of loss of residual renal function among new dialysis patients. J Am Soc Nephrol 11, 556-564 (2000).

13. He, L. et al. Rate of Decline of Residual Kidney Function Before and After the Start of Peritoneal Dialysis. Perit Dial Int 36, 334-339, https://doi.org/10.3747/pdi.2016.00024 (2016).

14. Berlanga, J. R., Marron, B., Reyero, A., Caramelo, C. \& Ortiz, A. Peritoneal dialysis retardation of progression of advanced renal failure. Perit Dial Int 22, 239-242 (2002).

15. Peritoneal Dialysis Adequacy Work, G. Clinical practice guidelines for peritoneal dialysis adequacy. Am J Kidney Dis 48 Suppl 1, S98-129, https://doi.org/10.1053/j.jkjd.2006.04.006 (2006).

16. Lo, W. K. et al. Guideline on targets for solute and fluid removal in adult patients on chronic peritoneal dialysis. Perit Dial Int 26, 520-522 (2006)

17. Sandrini, M. et al. Incremental peritoneal dialysis: a 10 year single-centre experience. J Nephrol 29, 871-879, https://doi.org/10.1007/ s40620-016-0344-z (2016).

18. Guest, S., Leypoldt, J. K., Cassin, M. \& Schreiber, M. Kinetic Modeling of Incremental Ambulatory Peritoneal Dialysis Exchanges. Perit Dial Int 37, 205-211, https://doi.org/10.3747/pdi.2016.00055 (2017).

19. Eknoyan, G. et al. Effect of dialysis dose and membrane flux in maintenance hemodialysis. N Engl J Med 347, 2010-2019, https://doi. org/10.1056/NEJMoa021583 (2002) 
20. Group, F. H. N. T. et al. In-center hemodialysis six times per week versus three times per week. N Engl J Med 363, 2287-2300, https:// doi.org/10.1056/NEJMoa1001593 (2010).

21. Chertow, G. M. et al. Long-Term Effects of Frequent In-Center Hemodialysis. J Am Soc Nephrol 27, 1830-1836, https://doi. org/10.1681/ASN.2015040426 (2016).

22. Yan, H. et al. Three Versus 4 Daily Exchanges and Residual Kidney Function Decline in Incident CAPD Patients: A Randomized Controlled Trial. Am J Kidney Dis 69, 506-513, https://doi.org/10.1053/j.ajkd.2016.08.019 (2017).

23. Daugirdas, J. T. et al. Effect of frequent hemodialysis on residual kidney function. Kidney Int 83, 949-958, https://doi.org/10.1038/ ki.2012.457 (2013).

24. Obi, Y. et al. Incremental Hemodialysis, Residual Kidney Function, and Mortality Risk in Incident Dialysis Patients: A Cohort Study. Am J Kidney Dis 68, 256-265, https://doi.org/10.1053/j.ajkd.2016.01.008 (2016).

25. Viglino, G., Neri, L. \& Barbieri, S. Incremental peritoneal dialysis: effects on the choice of dialysis modality, residual renal function and adequacy. Kidney Int Suppl, S52-55, https://doi.org/10.1038/sj.ki.5002601 (2008).

26. NKF-DOQI clinical practice guidelines for peritoneal dialysis adequacy. National Kidney Foundation. Am J Kidney Dis 30, S67-136 (1997).

27. Blake, P. G. et al. Clinical Practice Guidelines and Recommendations on Peritoneal Dialysis Adequacy 2011. Perit Dial Int 31, 218-239, https://doi.org/10.3747/pdi.2011.00026 (2011).

28. Garofalo, C. et al. Incremental dialysis in ESRD: systematic review and meta-analysis. J Nephrol, https://doi.org/10.1007/s40620018-00577-9 (2019).

29. Li, P. K. et al. ISPD Peritonitis Recommendations: 2016 Update on Prevention and Treatment. Perit Dial Int 36, 481-508, https://doi. org/10.3747/pdi.2016.00078 (2016).

30. Perl, J. et al. Changes in patient and technique survival over time among incident peritoneal dialysis patients in Canada. Clin J Am Soc Nephrol 7, 1145-1154, https://doi.org/10.2215/CJN.01480212 (2012).

31. Lee, S. et al. Technique failure in Korean incident peritoneal dialysis patients: a national population-based study. Kidney research and clinical practice 35, 245-251, https://doi.org/10.1016/j.krcp.2016.08.002 (2016).

32. Mehrotra, R., Nolph, K. D. \& Gotch, F. Early initiation of chronic dialysis: role of incremental dialysis. Perit Dial Int 17, 426-430 (1997).

33. Golper, T. A. Incremental dialysis. J Am Soc Nephrol 9, S107-111 (1998).

34. Ankawi, G. A., Woodcock, N. I., Jain, A. K., Garg, A. X. \& Blake, P. G. The Use of Incremental Peritoneal Dialysis in a Large Contemporary Peritoneal Dialysis Program. Can J Kidney Health Dis 3, 2054358116679131, https://doi. org/10.1177/2054358116679131 (2016).

35. Golper, T. A. \& Mehrotra, R. The intact nephron hypothesis in reverse: an argument to support incremental dialysis. Nephrol Dial Transplant 30, 1602-1604, https://doi.org/10.1093/ndt/gfv271 (2015).

36. Davies, S. J. Peritoneal dialysis in the patient with a failing renal allograft. Perit Dial Int 21(3), S280-284 (2001).

37. Madar, H. et al. Residual renal function in peritoneal dialysis after renal transplant failure. Perit Dial Int 30, 470-474, https://doi. org/10.3747/pdi.2009.00168 (2010).

38. Austin, P. C. The performance of different propensity score methods for estimating marginal hazard ratios. Stat Med 32, 2837-2849, https://doi.org/10.1002/sim.5705 (2013).

\section{Acknowledgements}

The authors gratefully acknowledge the assistance of Eun-Mi Yi, Yuri Kim, Baek-Soon Kim, Mi-young Song, and Suin Lee for the data collection; and the substantial contributions of the entire PD Unit of Seoul National University Hospital (physicians, nurses, and patients) that provide information to, and maintain, our database. The present study was funded by Fresenius Medical Care, Korea (SNUH Research No. 0620090493 and 0620161040). However, the funding body had no role in the design, implementation and the interpretation of the study.

\section{Author Contributions}

Conception or design was provided by Y.L., J.L. and K.O. Y.L., S.W.C., S.P. and J.L. were responsible for acquisition, analysis and interpretation of data. Drafting of the article or revising it was provided by H.R., H.L., D.K.K. and K.W.J. Intellectual content of critical importance to the work described was provided by Y.L., S.W.C., H.L., D.K.K., K.W.J., C.A. and K.O. Final approval of the version to be published was provided by Y.L., J.L. and K.O.

\section{Additional Information}

Competing Interests: The authors declare no competing interests.

Publisher's note: Springer Nature remains neutral with regard to jurisdictional claims in published maps and institutional affiliations.

(c) (i) Open Access This article is licensed under a Creative Commons Attribution 4.0 International cc) License, which permits use, sharing, adaptation, distribution and reproduction in any medium or format, as long as you give appropriate credit to the original author(s) and the source, provide a link to the Creative Commons license, and indicate if changes were made. The images or other third party material in this article are included in the article's Creative Commons license, unless indicated otherwise in a credit line to the material. If material is not included in the article's Creative Commons license and your intended use is not permitted by statutory regulation or exceeds the permitted use, you will need to obtain permission directly from the copyright holder. To view a copy of this license, visit http://creativecommons.org/licenses/by/4.0/.

(c) The Author(s) 2019 